\title{
The Experience-Based Safety Training System Using Vr Technology for Chemical Plant
}

\author{
Atsuko Nakai, Yuta Kaihata, Kazuhiko Suzuki \\ Center for Safe and Disaster-Resistant Society \\ Okayama University \\ Okayama, Japan
}

\begin{abstract}
In chemical plants, safety measures are needed in order to minimize the impact of severe accidents and natural disasters. At the same time, carrying out the education and training to workers the corresponding operation in nonstationary situation is essential. However, reproducing the nonstationary conditions to actual equipment or mock-up cannot be performed because it is dangerous. By using the virtual reality (VR) technology, we can build up a virtual chemical plant with lower cost compared to real plant. The operator can experience the fire and explosion accidents in the virtual space. Therefore, in this paper, we propose an experienced-based safety training system for implementing the education and training by using the non-stationary situation in the computer. This proposed system is linked with the dynamic plant simulator. A trainee can learn the correct operation through the simulated experience to prevent an accident. The safety awareness of workers will improve by experiential learning. The proposed system is useful for safety education in chemical plant.
\end{abstract}

\section{Keywords—safety education; training system; virtual reality}

\section{INTRODUCTION}

The chemical plant has a responsibility to provide and maintain a safe environment for people that live in such circumstances. As well-known many kinds of hazardous materials are under controlled in chemical facilities. Plant safety is provided through inherently safe design and various safeguards, such as instrumented systems, procedures, and training. Safety education/training is essentially important to prevent a severe accident. [1] However, in recent years, the retirement of a skillful operator is advancing in Japan. Because of this, the experts know-how and skill is lost. Skilled operators of superior technology has not been fully passed down to young engineers. Furthermore, immature young operator of the lack of experience is increasing. [2] If an accident occurs in a chemical plant, emergency shutdown is required rapidly. However, as revealed by accident at a nuclear power plant in Fukushima, after the plant has stopped safely, it has likely there resulted in serious damage from the error of the operator. [3] In an emergency, the operator is required to make a quick decision in order to prevent the expansion of the accident. But an untrained operator cannot respond enough to emergencies. Education/training of non-stationary operation for workers is needed. However, reproducing the nonstationary conditions to actual equipment or mock-up cannot be performed because it is dangerous. Also a company requires a huge cost to build a mock-up of the plant facilities for education. This paper will show the training system using virtual reality (VR) technology for chemical plants that we have developed. Figure 1 shows an outline of the training system. This system links the plant model within the VR environment from the process value of a dynamic plant simulator. Order to operate safely the safety device of various in chemical plants, operators have a responsible area of their own. There are two roles can be broadly divided into the plant operator. "Field man" is the role of operating the equipment in the field. Is the role that controls the refineries (control room) in the control center, "Board man". A trainee in the system can experience a cooperative work "Field man" and "Board man" in the similar environment of the real chemical plant.

This paper shows the safety training system using VR technology for chemical plant. The operator can experience the fire and explosion accidents in the virtual space. The safety awareness of workers will improve by experiential learning. Accidents don't happen the way they're supposed to be according to the manuals. We should consider what to do in case of an emergency.

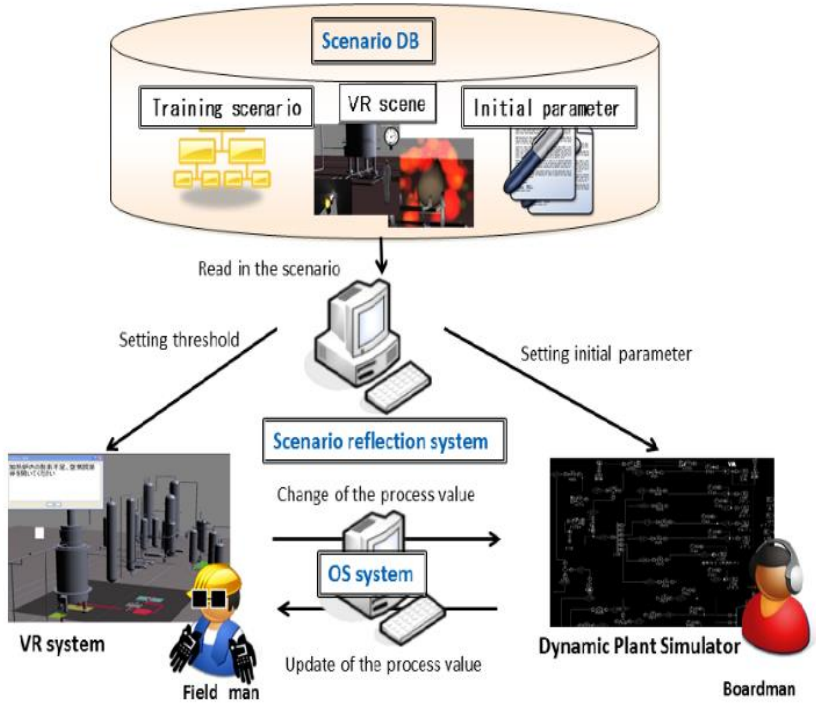

Fig. 1. Outline of the training system

\section{EXPERIENCE-BASED TRAINIG SySteM}

\section{A. Outline of the proposed system}

The system developed using virtual reality (VR) as new training method. VR is a technology that is artificially creating a sense of reality. [4] This technology is composed of a 
combination with computer graphics and acoustic efficiency. Thereby, the trainee can get feeling which is actually experiencing things in virtual plant. [5] Also dynamic plant simulator (DS) integrated dynamic simulation environment which reproduces behaviors resembling actual plant operations and provides for a realistic feeling. Therefore, DS has been used for operator training systems. [6][7] This proposed system is composed of a virtual plant that works in concurrence with the dynamic plant simulator(DS) in VR environment. In the result, it became possible to reflect the change of a process value correspond to time progress in the VR environment. [8] DS cannot reproduce severe accidents. Using VR technology, we can express the fire and explosion accidents. Operators/workers can learn the correct operation through the simulated experience to prevent an accident. In this study, the system in consideration of both field man and board man has developed. The board man inputs the process value to the dynamic simulator (DS) display. And the result is reflected in virtual plant. The field man operates the equipment by using the avatar in the Virtual plant through virtual plant screen. An event is qualitatively progress by reading animation in the virtual plant and reading the process value corresponding to the DS according to the amount of operation change. The training system is constructed by three subsystems "scenario database(DB)", "Operating Support(OS) System" and " Scenario reflection system", and two parts, "Virtual plant" and "Dynamic Simulator". The biggest difference between Web learning and VR environment, a trainee can reach out into the virtual world using VR technology. [9]

\section{B. The flow of training}

In order to convey training purposes, the system makes a training scenario to provide various information to the operator. Before training start, two or more training scenarios

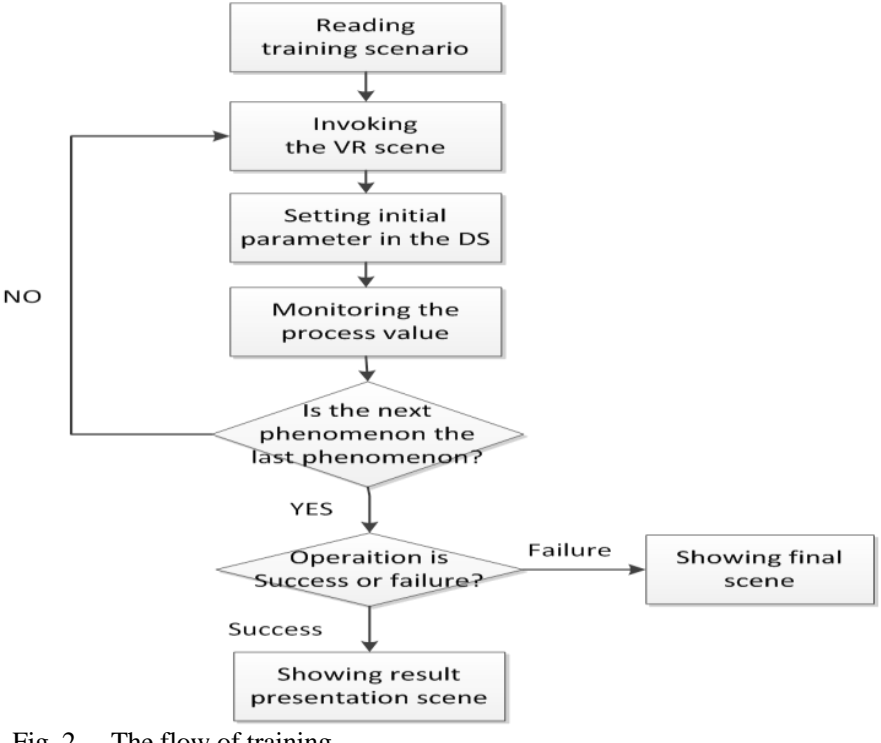

Fig. 2. The flow of training which described the branch criteria by threshold and the event contents are created. And, those are stored in the scenario DB. The flow of training is shown in Figure 2. Next, each VR scene cleated and initial parameters calculated in advance. The plant model in a virtual plant and the process value of DS are linked by OS system. Therefore, the change of the process value in a real plant is reflected to virtual plant. Scenario reflection system compares threshold which writing a select scenario and process value change of DS. When the process values meet the branch criteria, the event is progressing on the qualitatively of update VR scene and an initial parameter to correspond with the scene. In case the process value meets the branch criteria, the VR scene and the parameter of the process value are read by next VR plant and DS parameter. Hereby, it is possible for trainer to focus more to learn important event and to cut down the training time of the plant abnormality.

\section{Scenario database $(D B)$}

This chapter describes about the scenario data base in the training system. "Training scenario", "VR scene" and "initial parameters" are saved in scenario DB. And those are compatible with branch criteria so as to output each event. A training scenario is written over XML. Branch criteria and plant mode on the basis of flow rate, pressure and temperature, etc. are written in training scenarios. Scenario database has the VR scenes such as plant operation scene, the final scene and result presentation scene. VR scenes are created along the training scenario. Training is started from the plant scene. When the training starts, the display showed a plant scene. Trainer controls an avatar and makes a tour of inspection through the work site. When avatar approaches equipment like a valve in plant scene, equipment operation scene is displayed on the display. This training system updates corresponding scene over and over. This system shows the result presentation scene when a trainee success in early detection and accurate correspondence operation. Result presentation scene is displayed on the assembly operation that trainer went for. Conversely, this system shows a last phenomenon scene when a trainee fails in abnormal detection and correspondence operation. After that, the scene shift to the result presentation scene.

\section{Operation support(OS) system}

This system reflects the change of the process value into the passage of time in the VR environment by synchronizing the plant model in the VR environment with the process value of the plant simulator. Each process value of the plant simulator is stored in the Excel. The system the outputs process value as a CSV data form. And the VR environment reflects the behavior as a change of the process value of the equipment by storing the value in the array in the VR environment. Moreover, the equipment operation was synchronized with the simulator. When the equipment operation in the VR environment was done, the OS system writes the data in the CSV for the dynamic simulator operation through the Excel. 


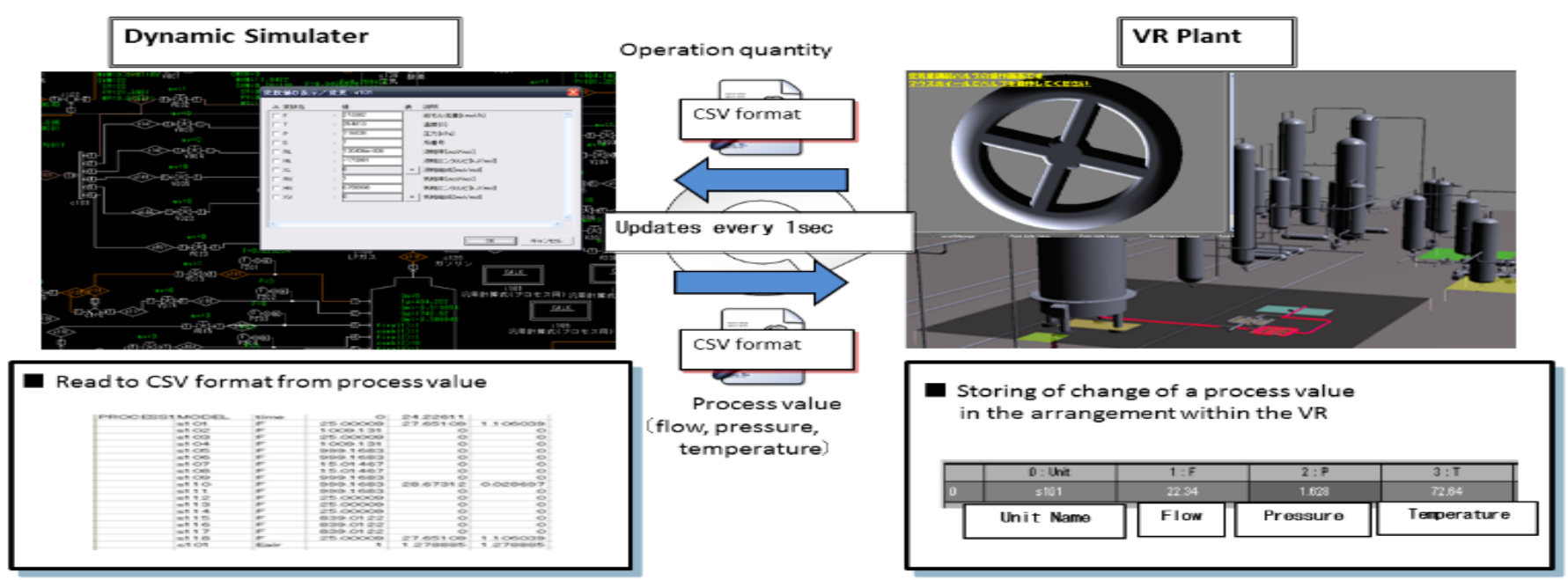

Fig. 3. Composition of OS system

A trainee can operate from the start/stop switch of the operation of valve and pump in the VR environment. Figure 3 shows the composition of OS system.

\section{E. Scenario reflection system}

In order to show a trainer an event continuously, it is necessary to output the initial parameter of VR scene and a process value for every event in accordance with the branch condition in a training scenario. The motion of the system from a training start is described along Figure 4. If a process value meets the branch criteria described by the training scenario by a trainer's equipment operation, a simulation will be stopped by suspending DS. Next, VR scene corresponding to the event 2 is read into the VR environment. The initial parameter of the process value corresponding to the event 2 is read into DS, and a simulation is resumed. Thus, the event divided by the threshold of the process value. The event can be continuously shown by making a simulation based on the initial parameter of the process value which differs from each event. [8]

\section{EXAMPLE OF APPLICATION}

\section{A. The application of the hydrodesulfurization process}

A hydrodesulfurization process is targeted in this system.

This process is the reaction with hydrogen separating and removing the sulfur content in a diesel using the metal catalyst of nickel, cobalt, molybdenum, etc. under high temperature and high pressure, and producing products, such as low kerosene of sulfur content, and light oil. The outline of a process is shown in Figure 5.

\section{B. Creation of VR scene based on the training scenario}

The training scenario that performs the abnormal detection and a correspondence operation is created. The VR scene express a heating furnace burner for this process. The system used malfunction scenarios from DS for education. [6] When a plant is in a stationary state, the air content in a heating furnace and the flame of the burner are kept normal. As for the flame of the burner, the size is kept at $1 / 2$ from $1 / 3$ to an ignition box ceiling in orange.

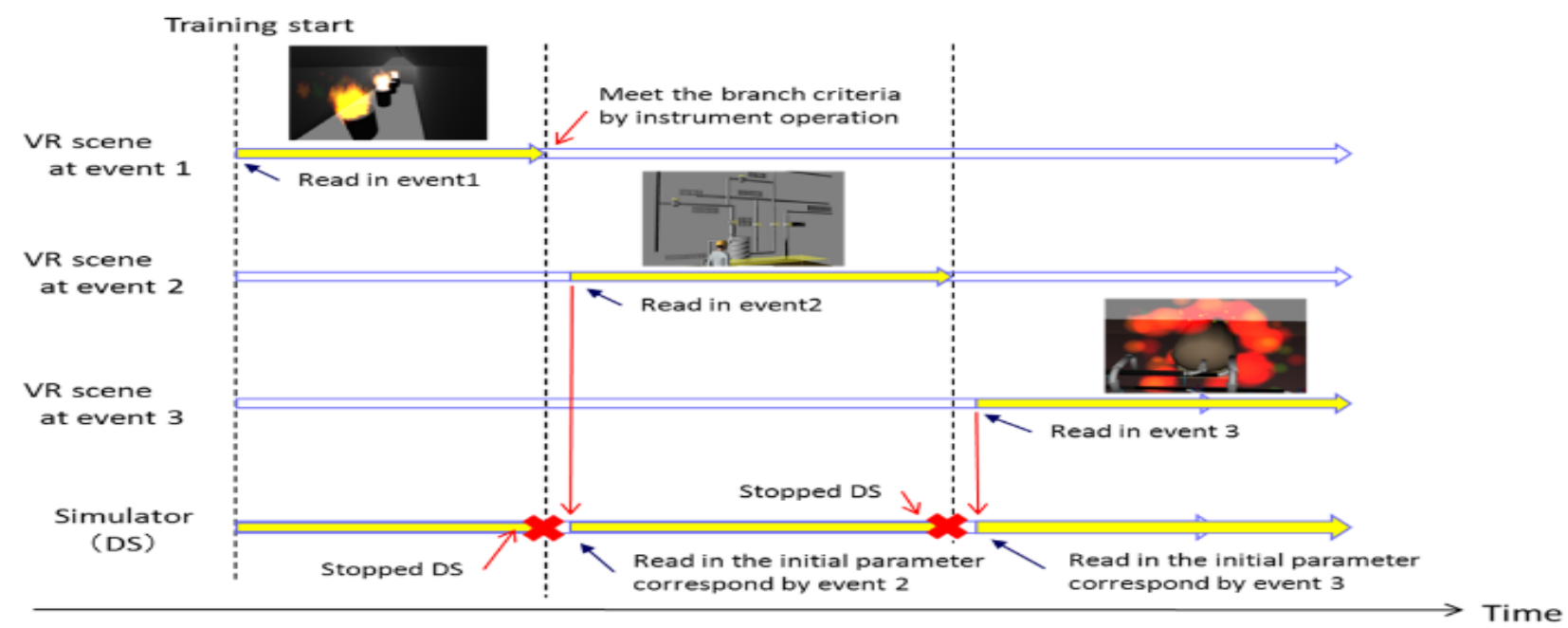

Fig. 4. Operation of the system in alignment with the time 
However, when supply of the air to fuel gas decreases, the content air in the heating furnace decreases.

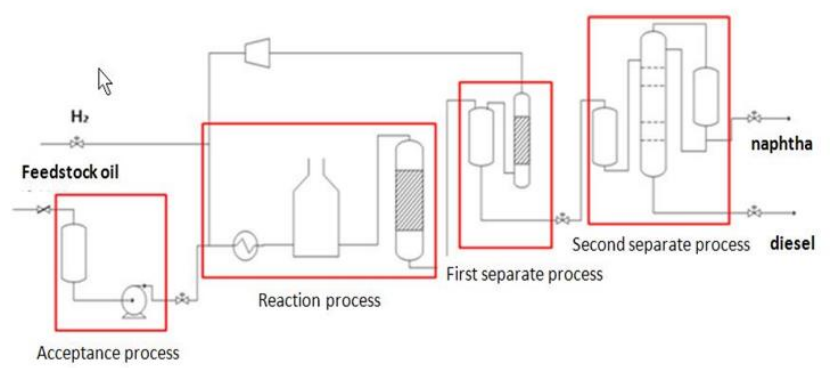

Fig. 5. The outline of a hydrodesulfurization process

Hereupon, the ignition box ceiling changes from a normal orange flame to yellow. Finally a burner is fired by negligence and there is fear of heating furnace explosion. The flame of the burner must be returned to normal color but in this situation it isn't so. When the fire of the burner goes out, the operator cut off the supply of fuel immediately. Next, in order to remove the fuel gas in the heating furnace, it is necessary to take steam purge 15 minutes or more. When the work is not done, combustible gas is full in a heating furnace. And it may be connected to a fire. The necessary scene is created based on the training scenario. The user interface is created so that trainer can check the information on the inside of a heating furnace and the instrumentation equipment in the display. As an accident which occurs in this example, the explosion by gas ignition in the heating furnace is assumed. So, on the last phenomenon scene, VR scene of the explosion, fire of the heating furnace as shown in Figure 6 is created.

\section{Integrate Dynamic plant simulator(DS)and VR scene}

When a training is carried out, the plant model and process value based on DS is linked by OS system. Accordingly, change of the process value of a real plant is reflected in VR plant. The situation of the link between DS and VR is shown in Figure 7. Pressure, temperature, and concentration are linked between the DS and the VR environment.

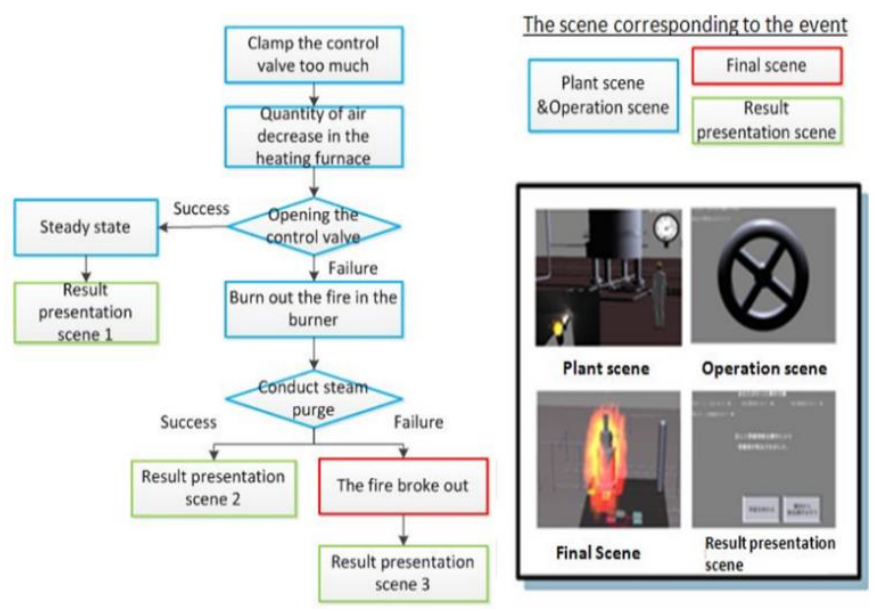

Fig. 6. Correspondence relation between training scenario and VR scene

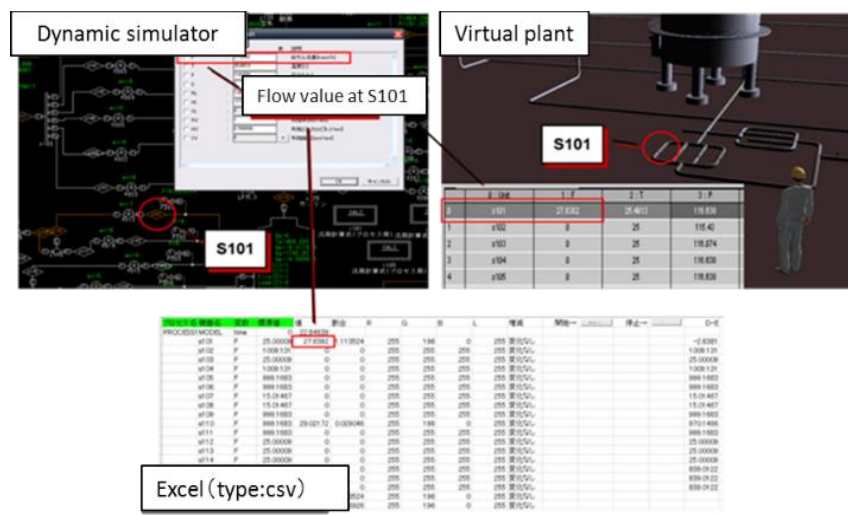

Fig. 7. The link of the process value of the DS and the VR environment

A VR event is developed by comparing the branch condition written in training scenario and the process value reflected in the VR environment. The following Figure 8 describes the event number 4 progress to the event number 5 in the training scenario.

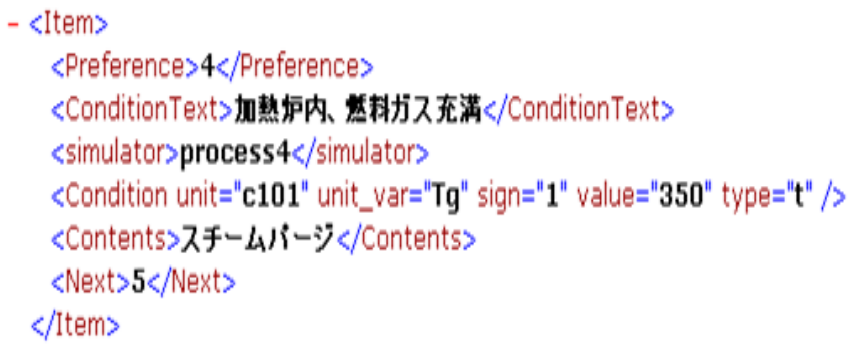

Fig. 8. The contents of the event number 4

If the number 4 event starts, the initial parameter value shows as process4. VR scene corresponding to process 4 describes in the <simulator>tag. And VR scene, bases on the $<$ Condition unit="c101"unit_var="Tg"sign="1"value="350"type="t"/>tag in the training scenario. This system supervises the process value $\mathrm{Tg}$ of "c101". "c101" shows a heating scene. If this condition is fulfilled by equipment operation into the VR environment, an event will progress to No. 5 from No. 4.

\section{Output of VR scene linked DS}

In this system, the process value has linked with the plant model in the VR environment by the above-mentioned OS system between the dynamic plant simulator. The scenario reflection system compares the branch conditions in the scenario by the process value stored in the array in the VR environment and outputs the corresponding scene. 


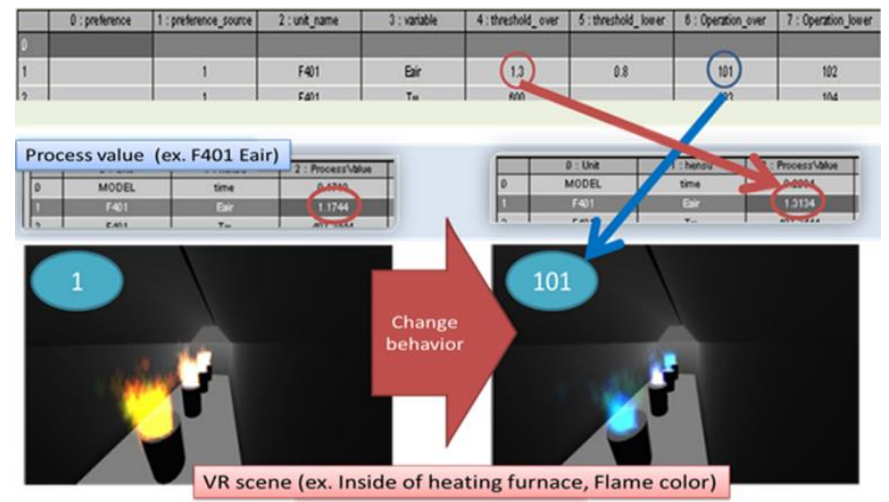

Fig. 9. Output of VR scene linked DS

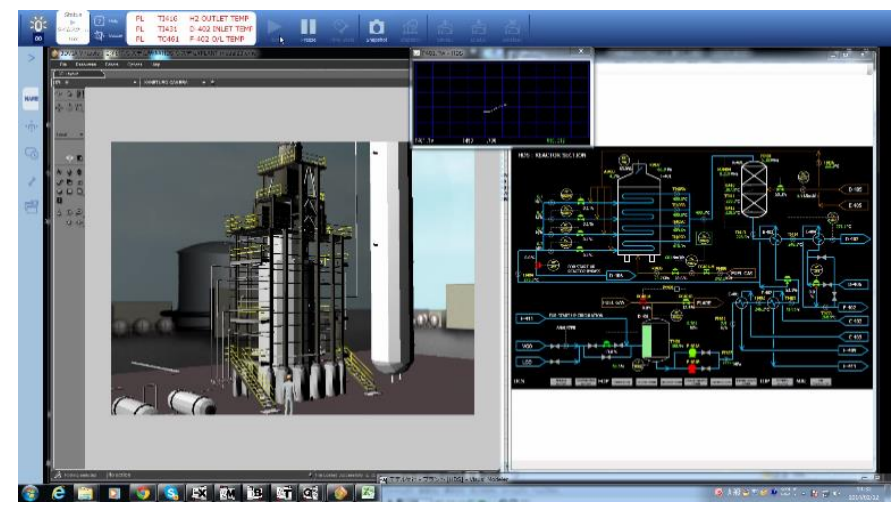

Fig. 10. The training system of the hydrodesulfurization process

Figure 9 describes the system can change the output based on the process value. Figure 10 shows the screen of the actual training system of the hydrodesulfurization process. In Figure 10, VR scene for "Field man" and DS panel for "Board man" are both in the same screen. The developed system can display VR scene and DS panel separate. Boardman and field man can learn operation another role in this training system.

\section{CONCLUSION}

In the chemical plant, rapid generation change of operators is progressing in Japan. The inheritance technology and accomplishments that have been worked in the chemical plant is essential to take advantage in order to extend the competitive production activities. When the accident/disaster happen, the corresponding operation without human error is required in the chemical plant. However, at present, sufficient training is not done for safety and cost issues. In this study, the Experiencebased safety training system using VR technology is developed. By using the VR technology, costs and location of the problem can be solved. The trainee can safely perform the training of the corresponding operation against the non-steady situation. In this system, virtual reality environment and dynamic plant simulator are integrated. A trainee in the system can experience a cooperative work "Field man " and "Board man" in a similar environment to the real chemical plant. The scenario reflection system compares the branch conditions in the scenario by the process value stored in the array in the VR environment and outputs the corresponding scene. Virtual plant can express the effects of natural disasters, not only reproduce the accident. Through training using a virtual plant, a trainee can experience the hazardous conditions that cannot be experienced in real. This training is useful for safety education/training for the workers who have no experience. In future this system will be extended to accommodate operation of two people at the same time. After the experience-based learning in virtual plant, the operator should review the corresponding operation to the accident. We will add a relearning part into this system.

The proposed system will lead to the improvement of the safety awareness of day-to-day operations, consisting of knowledge, skill, sensibility, "total power".

\section{REFERENCES}

[1] S. Jürgen, "Process and Plant Safety - Research \& Education Strategy to Keep Long Term Competences", Chemical Engineering Transactions, vol.31, 2013.

[2] T. Ogawa, "The security measure subject of the latest chemical factory", SCAS NEWS 2003 pp.1-2

[3] Investigation Committee on the Accident at Fukushima Nuclear Power Stations of Tokyo Electric Power Company, Executive Summary of the Final Report, 23 July 2012.

[4] D. Schofield, "Mass Effect: A Chemical Engineering Education Application of Virtual Reality Simulator Technology", MERLOT Journal of Online Learning and Teaching vol. 8, No. 1, March 2012.

[5] A.Wasfy, T. Wasfy, A. Noor, "Intelligent virtual environment for process training”, Advances in Engineering Software, No. 35, 2004.

[6] G. Fukano, K. Yokoyama, Y. Yahata, "MIRROR PLANT On-line Plant Simulator and its Applications", Yokogawa Technical Report English Edition ,vol.56 No.1, 2013 pp. 11- 14.

[7] S. Nazir, S. Colombo, D. Manca, "Minimizing the Risk in the Process Industry by Using a Plant Simulator: a Novel Approach", Chemical Engineering Transactions, vol.32, 2013 pp.109-114.

[8] K. Yamamoto, A. Nakai, K. Suzuki, "Development of Experiencedbased Training System combined with Process Dynamic Simulation", Asia Pacific Symposium on Safety 2013, October 2013.

[9] C. Norton, I. Cameron, C. Crosthwaite, N. Balliu, M. Tade, D. Shallcross, A. Hoadley, G. Barton, J. Kavanagh, "Development and deployment of an immersive learning environment for enhancing process systems engineering concepts", Education for Chemical Engineers,vol. 3, 2, Decwmber 2008 pp.75-83. 